\title{
Control of the Metal Rolling Process: A Multidimensional System Approach
}

\author{
S. Foda \\ Ryerson University

\section{P. Agathoklis} \\ University of Victoria
}

digital.library.ryerson.ca/object/268

\section{Please Cite:}

Foda, S., \& Agathoklis, P. (1989). Control of the metal rolling process: A multidimensional system approach. Proceedings of the 1989 American Control Conference, 2405-2410.

doi:10.23919/ACC.1989.4790593

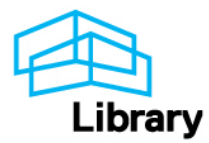




\section{Control of the Metal Rolling Process: A Multidimensional System Approach}

\author{
S. Foda \\ Department of Electrical Engineering \\ Ryerson Polytechnical Institute \\ 350 Victoria Street, Toronto \\ Canada M5B 2K3
}

\begin{abstract}
The metal-rofling process is modelled as a delay differential system with noncommensurate delays. Stability conditions for delay diferential systems have been recently developed using asymptotic stability tests and the Lyapmov approach. These results are applied to the stability analysis and stabilisation of the metal rolling process. Conditions on the gains of finite dimensional controllers such as proportional plus derfvative controllers are developed to ensure the stability of the controlled process against delay variations.
\end{abstract}

Keywords: Metal rolling, multipass processes, delay differential systems, multidimensional systems.

\section{Notation}

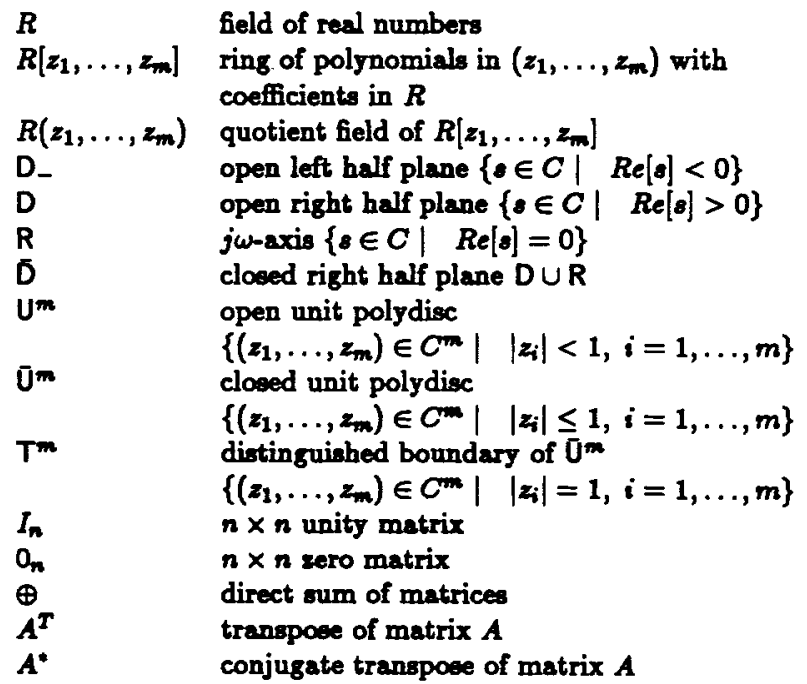

\section{Introduction}

Metal rolling is a common plastic working technique in which deformation takes place between two rolls with parallel axes revolving in opposite directions as shown in Fig. 1. In practice, the stock is passed through a series of rolls for successive reductions which requires more investment in equipment [1]. A more economical practice is to use a single two high stand; a process often called 'cogging' [2].

\author{
P. Agathoklis \\ Department of Electrical and Computer \\ Engineering, University of Victoria \\ P.O. Box 1700, Victoria, B.C. \\ Canada V8W $2 Y 2$
}

In this paper, the cogging process is considered as a multipass process. Multipass processes represent a class of dynamical systems that operate in repetitive cycles. Each cycle of operation is called a pass and the interaction between the outputs generated during successive cycles of operation is called pass interaction. Examples of such processes, besides the metal rolling, are metal cutting processes, binary distillation columns, liquid/liquid heat exchangers, automatic ploughing, vehicle convoys, and the standard multipass process 'verticallysteered longwall cool-cutting machine' $[3,4,5]$. The approach taken in this paper is 80 general to be applicable to any of the above mentioned processes as well.

The stability of multipass processes has been examined by Edwards [2] where the multipass process is converted into an infinite-length single-pass process described by a functional differential equation and then the standard inverse-Nyquist stability criteria is applied. This approach has been examined by Owens [6,7] who showed that it neglects disturbances at the beginning of each pass. Therefore, it is natural to introduce pass delay and to discuss the stability of multipass processes using multidimensional system stability theory. In two more recent publications Boland and Owens [8] and Edwards and Owens [5] showed that for discretized multipass processes two-dimensional (2-D) bounded-input bounded-output (BIBO) stability is equivalent to what is known as the stability along the pass [2]. An analogous result for differential multipass processes has been presented in Foda and Agathoklis [9]. In [10] the coefficient assignment for 2-D discrete systems has been applied to design a tracking controller for the metal rolling process.

Differential multipass processes have been recently studied using delay differential system models [11]. Analytic tests for asymptotic stability and stability independent of delay (i.o.d.) for delay differential systems are available $[12,13,14]$. Also, sufficient conditions for the stability of delay differential systems using the Lyapunov approach have been developed by Agathoklis and Foda $[15,9]$. These stability results are used to determine the constraints on the gains of the finite dimensional controllers such as proportional plus derivative $(\mathrm{PD})$ controllers in order that the controlled metal rolling process would be stable against delay variations. 
The paper is organised as follows. The next section contains background material and results pertinent to delay differential and multipass systems. In Section 3, the metal rolling is modelled as a delay differential system with two noncommensurate delays, namely the pass delay and the gauge sensor measurement delay. In Section 4, the stability of the metal rolling process is considered. Proportional plus derivative (PD) controllers are designed for the controlled metal rolling process using the asymptotic stability i.o.d. criteria for delay differential systems. It is shown that this criteria, which is stronger than the asymptotic stability criteria for delay differential systems, is more appropriate, taking into consideration the nature of the delays involved. Discussion and some concluding remarks follow in Section 6.

\section{Delay Differential Systems}

In this section, delay differential system models and their stability are discussed. Our interest in this type of multidimensional systems is derived from the fact that the metal rolling process discussed in the next section is a differential multipass process $[11,6,7]$ and such processes can be modelled via delay differential equations.

An autonomous delay differential system with $m$ noncommensurate delays is represented by a functional differential equation of the form

$$
\begin{aligned}
& \sum_{i=0}^{n_{m+1}} \sum_{j=0}^{n_{1}} \ldots \sum_{j_{m}=0}^{n_{m}} c_{i j_{1} \ldots . . j m} \frac{d}{d t^{i}} y\left(t-j h_{1}-j_{i} h_{2}-\ldots-j_{m} h_{m}\right) \\
& +\frac{d^{m-1}}{d^{m_{m+1}}} y(t)=0
\end{aligned}
$$

where $h_{1}, \ldots, h_{m}$ are the noncommensurate delays. Delays $h_{1}, \ldots, h_{m}$ are called noncommensurate if there exist no integers $l_{1}, \ldots, l_{m}$ ( not all of them zero) such that $\sum_{i=1}^{m} l_{i} h_{i}=0$. Eq. $(1)$ is called commensurate if $m=1$.

The characteristic polynomial associated with this delay differential system would be a multivariate of the form

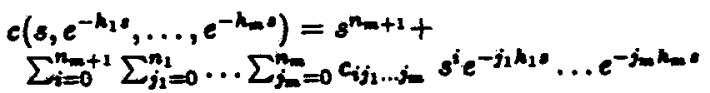

Letting $z_{i} \triangleq e^{-\lambda_{i} \theta}, i=1, \ldots, m$, i.e. $z_{i}$ is a left shift operator of duration $h_{i}$, the characteristic polynomial would be

$$
\begin{aligned}
& c\left(s, z_{1}, \ldots, z_{m}\right)=s^{n_{m+1}}+ \\
& \sum_{i=0}^{n_{m+1}} \sum_{j_{1}=0}^{n_{1}} \ldots \sum_{j=0}^{n_{m}} c_{i j_{1} . . j_{m}} s^{i} z_{1}^{j_{1}} \ldots z_{m}
\end{aligned}
$$

An autonomous delay differential system of the commensurate type can be modelled via the following 2-D state space model $[11,16]$

$$
\left[\begin{array}{c}
x_{1}(t+h) \\
\dot{x}_{2}(t)
\end{array}\right]=\left[\begin{array}{cc}
A_{1} & A_{2} \\
A_{3} & A_{4}
\end{array}\right]\left[\begin{array}{l}
x_{1}(t) \\
x_{2}(t)
\end{array}\right]
$$

where $x_{1} \in R^{n_{1}}$ denotes the delay state vector, $x_{2} \in$ $x^{n}$ denotes the differential state vector, and $h$ denotes the pass delay for a differential multipass process. The characteristic polynomial of this 2-D state space model is defined as

$$
c(s, z) \triangleq \operatorname{det}\left[\begin{array}{cc}
I_{n_{1}}-z A_{1} & -z A_{2} \\
-A_{3} & s I_{n_{2}}-A_{4}
\end{array}\right]
$$

which can be written in in the form of (3). This 2-D state space model realizes both neutral ( $c_{n_{2} j} \neq 0$, for some $\left.j \in\left[1, n_{1}\right]\right)$ and retarded $\left(c_{n_{2} j}=0, \forall j \in\left[1, n_{1}\right]\right)$ delay differential systems.

Also, a delay differential system with $m$ noncommensurate delays may be modelled by the following generalized linear system model $[17,18]$

$$
\begin{array}{r}
D\left(z_{1}, \ldots, z_{m}\right) \dot{x}(t)=A\left(z_{1}, \ldots, z_{m}\right) x(t)+ \\
B\left(z_{1}, \ldots, z_{m}\right) u(t)
\end{array}
$$

where $A, D \in R^{n \times m}\left[z_{1}, \ldots, z_{m}\right]$ and $B \in R^{m \times r}\left[z_{1}, \ldots, z_{m}\right]$. $z_{i}, i=1, \ldots, m$ are delay operators of duration $h_{i}, i=$ $1, m$. Note that $R\left[z_{1}, \ldots, z_{m}\right]$ is a commutative ring in general and for $m=1$, i.e. the commensurate case, it is also a principal ideal domain (p.i.d).

Definition 1 [19] A matrix $D\left(z_{1}, \ldots, z_{m}\right)$ $\in R^{n \times n}\left[z_{1}, \ldots, z_{m}\right]$ is atomic at zero if $D(0)$ is nonsingular over $\boldsymbol{R}$.

Atomicity at sero is atways assumed for $D$ in this paper. Let $\bar{U}_{f}$ be the clowed polydiak $\mathbf{O}_{f}=\left\{\left(z_{1}, \ldots, z_{m}\right) \in\right.$ $\left.C^{m}|| z_{i} \mid \leq 1+\delta, i=1, \ldots, m\right\}$, then formal stability of $D$ is defined as follows:

Definition $2[17] D\left(z_{1}, \ldots, z_{m}\right)$ is called formally stable if $\operatorname{det}\left[D\left(z_{1}, \ldots, z_{m}\right)\right] \neq 0$ for all $\left(z_{1}, \ldots, z_{m}\right) \in 0_{k}$ for some $\delta>0$.

Remark: If $D$ is atomic at sero and formally stable, then it is invertible over $0^{\mathbf{m}}$ and Eq. (7) becomes

$$
\dot{x}(t)=F\left(z_{1}, \ldots, z_{m}\right) x(t)+G\left(z_{1}, \ldots, z_{m}\right) u(t)
$$

where $F=D^{-1} A$ and $G=D^{-1} B$ are in general over $R\left(z_{1}, \ldots, z_{n}\right)$.

The stability of delay differential systems has been studied via the investigation of the zero sets of the chatacteristic polynomial $[12,13,14]$ and using $1-D$ frequency dependent Lyapunor equations. Also, $m$-D constant parameter Lyapunov equations of a special form, that combines both known forms of the Lyapunov equation, have been recently derived $[15,20]$ for the stability analysis of delay differential systems. First let $h=\max h_{i}, i=$ $1, \ldots, m$ and let $B$ denote the Banach space of continuous functions from $[-h, 0]$ into $R$ equipped with the sup norm

$$
\|\psi\|=\sup _{\sigma \in[-\lambda, 0]}|\psi(\sigma)|, \text { for any } \psi \in B
$$

Also let $y_{t} \in \mathrm{B}$ denote the function segment defined by

$$
y_{t}(\sigma)=y(t+\sigma), \quad \sigma \in[-h, 0]
$$

Consider the autonomous delay differential system given by Eq. (1) which is usually considered with the 
initial condition

$$
y_{0}(t)=\psi(t), \quad t \in[-h, 0], \text { where } \psi \in B
$$

Definition 3 [21] The delay differential system (1) is said to be asymptotically stable if $\exists M, \gamma>0$ such that for each $\psi \in B$ the solution $y(t)$ of (1) with $y_{0}=\psi$ satisfies

$$
\|y(t)\| \leq M|\psi| e^{-\gamma t}, \quad \forall t \geq 0
$$

and is said to be asymptotically stable independent of delay (i.o.d.) if (9) holds for all $h_{i} \geq 0, i=$ $1, \ldots, m$.

The following theorem states the necessary and suffcient conditions for the asymptotic stability of (1).

Theorem 1 [22] The delay differential system described by Eq. (1) is asymptotically stable if and only if

$$
c\left(s, e^{-h_{1}}, \ldots, e^{-h_{m}}\right) \neq 0, \quad R e s \geq 0,
$$

and asymptotically stable independent of delay (i.o.d.) if and only if (10) holds $\forall h_{i} \geq 0, i=1, \ldots, m$.

Corollary 1 The delay differential system (7) is asymptotically stable

$$
\operatorname{det}\left[s I-F\left(e^{-s h_{1}}, \ldots, e^{-s h_{m}}\right)\right] \neq 0, \forall s \in \bar{D} \text {, }
$$

and asymptotically stable independent of delay (i.o.d.) if and only if (11) holds $\forall h_{i} \geq 0, i=1, \ldots, m$.

The following theorem provides an analytic test for i.o.d. asymptotic stability.

Theorem 2 [12] The delay differential system (1) is i.o.d. asymptotically stable if and only if

(i) $\quad c(s, 1) \neq 0, \quad s \in D$

(ii) $c(s,-1) \neq 0, \quad s \in R, \quad(s \neq 0)$

(iii) $(1+s T)^{n_{1}} c\left(s, \frac{1-s T}{1+s T}\right) \neq 0, \quad s \in R, \quad \forall T>q 14$

A stronger notion of stability for delay differential systems called pointwise asymptotic stability has been defined using the characteristic multivariate $c\left(s, z_{1}, \ldots, z_{m}\right)$ as follows.

Definition $4[23,24]$ A delay differential system with characteristic polynomial $c\left(s, z_{1}, \ldots, z_{m}\right)$ is said to be pointwise asymptotically stable if and only if

$$
c\left(s, z_{1}, \ldots, z_{m}\right) \neq 0, \quad \forall\left(s, z_{1}, \ldots, z_{m}\right) \in \bar{D} \times \bar{U}^{m}(15)
$$

Therefore the delay differential system described by Eq. (7) is pointwise asymptotically stable if and only if

$$
\begin{aligned}
\operatorname{det}\left[s I-F\left(z_{1}, \ldots, z_{m}\right)\right] & \neq 0, \\
\forall\left(s, z_{1}, \ldots, z_{m}\right) & \in \bar{D} \times \bar{U}^{m}
\end{aligned}
$$

The stability of the 2-D state space model (4) has been studied via a constant parameter 2-D Lyapunov Equation as follows $[15,20]$. Let $W=W_{1} \oplus W_{2}$ and $Q$ be positive definite symmetric (p.d.s.) matrices over $R$. Then the proposed 2-D Lyapunov equation for delay differential systems is

$$
A^{T} W^{0,1}+W^{0,1} A+A^{T} W^{1,0} A-W^{1,0}=-Q
$$

where $W^{1,0} \triangleq W_{1} \oplus 0_{n_{2}}, W^{0,1} \triangleq 0_{n_{1}} \oplus W_{2}$, and $A \triangleq$ $\left[\begin{array}{cc}A_{1} & A_{2} \\ A_{3} & A_{4}\end{array}\right]$

It is clear that this proposed Lyapunov equation combines known discrete and continuous forms of the known Lyapunov equations. The following theorem gives a sufficient 2-D stability condition using this proposed Lyapunov equation.

Theorem 3 [15] The delay differential system (4) is pointwise asymptotically stable if there exist p.d.s. matrices $W_{1}, W_{2}$, and $Q$ such that the 2-D Lyapunov equation (17) is satisfied where $W=W_{1} \oplus W_{2}$ and $A$ is as given before.

\section{The Metal Rolling Process}

In this section the metal rolling process is described and modelled as a delay differential system of the noncommensurate type. Fig. 2 depicts the physical diagram of

the metal rolling process. The dynamics of this process are given by [5].

$$
\begin{gathered}
y_{o}(t)=\frac{1 / M}{p^{2}+2 \xi \omega_{o} p+\omega_{0}^{2}}\left[-\frac{k_{a} \lambda}{\lambda_{2}} y_{d}(t)+\frac{\lambda^{2}}{\lambda_{2}} y_{i}(t)\right] \\
+\frac{\lambda}{\lambda_{1}} y_{i}(t)
\end{gathered}
$$

where $p$ denotes the differentiation operator $d / d t$, $y_{0}(t)$ is the actual roll-gap thickness,

$y_{i}(t)$ is the thickness of the incoming metal strip,

$y_{d}(t)$ is the desired value of motor deflection from the unstressed position,

$M$ is the lumped mass of the roll-gap adjusting mechanism,

$\lambda_{1}$ is the stiffness of the adjusting mechanism spring,

$\lambda_{2}$ is the hardness of the metal strip,

$\lambda=\lambda_{1} \lambda_{2} /\left(\lambda_{1}+\lambda_{2}\right)$ is the composite stiffness of the metal strip and the roll mechanism,

$\omega_{0}=\left[\left(k_{a}+\lambda\right) / M\right]^{1 / 2}, \xi=k_{b} /\left(2 \omega_{0} M\right)$ are the angular natural frequency and the damping ratio of the local servo,

and $k_{a}, k_{b}$ are the proportional and derivative gains of the servo controller.

The work strip can be passed back and forth through a reversing stand (cf. Fig. 3) which requires extra power [1]. Therefore, it is assumed that the strip is passed repeatedly through a non-reversing single stand where the roll-gap is reduced for each pass, a process often called 'cogging' [2]. Nonetheless, this process is slow 
and has a variable pass delay since the stock is usually passed over the top of rolls.

The thickness of the incoming strip can be related to the actual roll-gap thickness by the following interpass equation

$$
y_{i}(t)=y_{0}\left(t-h_{1}\right)
$$

where the delay $h_{1}$ denotes the pass delay and it may be related to $L$, the length of the metal strip which varies from pass to pass. Eq. (19) will be referred to as the pass interaction equation.

The gauge thickness is usually controlled via a proportional feedback control of the form

$$
y_{d}(t)=-k_{e}\left[y_{r}(t)-y_{0}\left(t-h_{2}\right)\right]
$$

where $k_{c}$ is the loop gain and $y_{r}(t)$ is the adjustable reference setting for the desired strip thickness. The delay $h_{2}$ is the output sensor measurement delay which is given by $h_{2}=X / v(t)$ where, as shown in Fis. 3, $X$ is the distance between the roll-gap and the output sensor and $v(t)$ is the velocity of the metal strip which may also vary from pass to pass. Hence, the controlled metal rolling process is described by the delay differential equations (19-20) and

$$
\begin{gathered}
y_{\circ}(t)=\frac{1 / M}{p^{2}+2 \xi \omega_{0} p+\omega_{0}^{2}}\left[-c_{1} k_{\infty} y_{d}(t)+c_{2} y_{i}(t)\right] \\
+c_{s} v_{i}(t)
\end{gathered}
$$

where $c_{1} \triangleq \lambda / \lambda_{2}, c_{2} \triangleq \lambda c_{1}$, and $c_{3} \triangleq \lambda / \lambda_{1}$. From Fq. (21)

$$
\begin{aligned}
&\left(p^{2}+2 \xi \omega_{0} p+\omega_{0}^{2}\right)\left[y_{0}(t)-c_{3} y_{i}(t)\right]=-\frac{c_{1} k_{0}}{M} y_{d}(t)+ \\
& \frac{c_{2}}{M} y_{i}(t) \quad(22)
\end{aligned}
$$

Substituting in the above equation for $y_{i}(t)$ and $y_{d}(t)$ using Eqns. (19) and (20), respectively, we get

$$
\begin{array}{r}
\left(p^{2}+2 \xi \omega_{0} p+\omega_{0}^{2}\right)\left[y_{0}(t)-c_{s} y_{0}\left(t-h_{1}\right)\right]= \\
\frac{c_{1} k_{c} k_{c}}{M}\left[y_{h}(t)-y_{0}\left(t-h_{2}\right)\right]+\frac{c_{2}}{M} y_{0}\left(t-h_{1}\right)
\end{array}
$$

Thus the controlled metal rolling process can be modelled by the following forced delay differential equation

$$
\begin{aligned}
\bar{y}_{0}(t)+2 \xi \omega_{0} \dot{y}_{0}(t)+\omega_{0}^{2} y_{0}(t)-c_{3} \dot{y}_{0}\left(t-h_{1}\right) \\
-2 \xi \omega_{0} c_{3} \dot{y}_{0}\left(t-h_{1}\right)-\left(\omega_{0}^{2} c_{3}+\frac{c_{2}}{M}\right) y_{0}\left(t-h_{1}\right)+ \\
\quad \frac{c_{1} k_{a} k_{c}}{M} y_{0}\left(t-h_{2}\right)=\frac{c_{1} k_{a} k_{c}}{M} y_{r}(t)
\end{aligned}
$$

where $\omega_{0}^{2}=\left(k_{a}+\lambda\right) / M$ and $2 \xi \omega_{0}=k_{b} / M$. This is a delay differential system of the neutral type with two noncommensurate delays $h_{1}$ and $h_{2}$. The characteristic multivariate associated with this system can be written in the form

$$
c\left(s, z_{1}, z_{2}\right)=s^{2}+\sum_{i=0}^{2} \sum_{j_{1}=0}^{1} \sum_{j_{2}=0}^{1} c_{i j_{1} j_{2}} s^{i} z_{1}^{j_{1}} z_{2}^{2}
$$

Given this delay differential equation, it is possible to write it in a generalized system model over $R\left[z_{1}, z_{2}\right]$ as follows. Let $z_{1,2}$ be the shift operators defined by $z_{i} y_{0}(t) \triangleq y_{0}\left(t-h_{i}\right), i=1,2$. Also, define $x_{1}(t) \triangleq y_{0}(t)$, $x_{2}(t) \triangleq \dot{y}_{0}(t)$ and $x^{T}(t) \triangleq\left[x_{1}(t), x_{2}(t)\right]$. Then Eq. (24) can be modelled by

$$
D\left(z_{1}, z_{2}\right) \dot{x}(t)=A\left(z_{1}, z_{2}\right) x(t)+B\left(z_{1}, z_{2}\right) y_{r}(t)
$$

where $A, B$, and $D$ are over the ring $R\left[z_{1}, z_{2}\right]$ and

$$
\begin{aligned}
& A\left(z_{1}, z_{2}\right)=\left[\begin{array}{cc}
0 & 1 \\
a_{21}\left(z_{1}, z_{2}\right) & a_{z 2}\left(z_{1}, z_{2}\right)
\end{array}\right] \text {, where } \\
& a_{21}\left(z_{1}, z_{2}\right)=\omega_{0}^{2}-\left(\frac{c_{2}}{M}+\omega_{0}^{2} c_{3}\right) z_{1} \\
& +\left(c_{1} k_{a} k_{c} / M\right) z_{2} \\
& \text { and } a_{22}\left(z_{1}, z_{2}\right)=-2 \xi \omega_{0}\left(1-c_{3} z_{1}\right) \\
& B\left(z_{1}, z_{2}\right)=\left[\begin{array}{c}
0 \\
c_{1} k_{0} k_{*} / M
\end{array}\right],
\end{aligned}
$$

and

$$
D\left(z_{1}, z_{2}\right)=\left[\begin{array}{cc}
1 & 0 \\
0 & \left(1-c_{3} z_{1}\right)
\end{array}\right] .
$$

It is clear that $D\left(x_{1}, z_{2}\right)$ is atomic at rero and that it is formally stable since $c_{3}=\lambda_{2} /\left(\lambda_{1}+\lambda_{2}\right)<1$. Hence, $D\left(z_{1}, z_{2}\right)$ is invertible over the closed unit bidisc and $\mathrm{Eq}$. (26) can be written as

$$
\dot{s}(t)=F\left(z_{1}, z_{2}\right) x(t)+G\left(z_{1}, z_{2}\right) y_{h}(t)
$$

where

$$
\begin{aligned}
F\left(z_{1}, z_{2}\right)= & {\left[\begin{array}{ll}
0 & 1 \\
f_{1} & f_{2}
\end{array}\right] \text { and } G\left(z_{1}, z_{2}\right)=\left[\begin{array}{l}
0 \\
g
\end{array}\right], } \\
\text { with } f_{1}= & {\left[\omega_{0}^{2}-\left(\frac{c_{2}}{M}+\omega_{0}^{2} c_{3}\right) z_{1}\right.} \\
& \left.+\frac{c_{1} k_{3} k_{c}}{M} z_{2}\right] /\left(1-c_{3} z_{1}\right), \\
f_{2}= & -2 \xi \omega_{0}, \text { and } g=\frac{c_{1} k_{0} k_{e}}{M\left(1-c_{3} z_{1}\right)} .
\end{aligned}
$$

If the pass delay is an integer multiple of the output sensor measurement delay or the measurement delay can be neglected, the above models would be commensurate. Another case that results in a commensurate model is the so-called previous pass control [5] for which $h_{2}=h_{1}$. For example, if $h_{2}$ is neglected the metal rolling process dynamics would be described by the state space model

$$
\dot{x}(t)=F(z) x(t)+G(z) y_{r}(t)
$$

where $\left.f_{1}=\left[\omega_{0}^{2}+\frac{c_{1} k_{a} k_{c}}{M}-\left(\frac{c_{2}}{M}+\omega_{0}^{2} c_{3}\right) z\right] /\left(1-c_{3} z\right)\right]$, $f_{2}$, and $g$ are as before.

\section{Stability Analysis and Stabi- lization}

In this section the stability of the metal rolling process 
is analysed as a delay differential system. The objective is to calculate bounds for the PD controller gains $k_{a}, k_{b}$, and $k_{c}$ to guarantee the stability of the controlled process described by the delay differential equation (24). $h_{1}$ and $h_{2}$ are not constant in general and therefore, asymptotic stability in delay intervals and asymptotic stability i.o.d. should be considered (cf. Definition 3). However, it is very difficult in general to check any of these stability conditions for the noncommensurate case and, therefore, one can consider pointwise asymptotic tability using Eq. (16). Condition (16) can be further simplified due to the fact that the regularity of $F\left(z_{1}, z_{2}\right)$ in Eq. (27) on the closed unit bidisc $\bar{U}^{2}$ implies that the eigenvalues $\lambda_{i}$ of $F\left(z_{1}, z_{2}\right)$ are regular functions of $\left(z_{1}, z_{2}\right)$. Thus if $R e \lambda_{i}>0$ for some $\left(z_{1}^{0}, z_{2}^{0}\right) \in \bar{U}^{2}$, it must be true that Re $\lambda_{i}>0 \quad \forall\left(z_{1}, z_{2}\right)$ in an open neighbourhood of $\left(z_{1}^{0}, z_{2}^{0}\right)$. Therefore, instability of the multipass process in the pointwise asymptotic stability sense can be detected by checking the eigenvalues of $F\left(z_{1}, z_{2}\right)$ on $T^{2}$. This can be carried out using a suitably fine partition of $\mathrm{T}^{2}$ which is a finite computation. Furthermore, the testing of the eigenvalues of $F\left(z_{1}, z_{2}\right)$ can be done by applying existing stability tests for polynomials with complex coefficients [25] to the characteristic polynomial of $F\left(z_{1}, z_{2}\right)$.

The stability analysis can be further simplified by considering the case where the sensor delay is neglected. This leads to a commensurate model for which asymptotic stability can be checked using the analytic stability test provided by Theorem 2. Assuming zero reference input, the delay differential equation (24) becomes

$$
\begin{aligned}
& \dot{y}_{0}(t)+2 \xi \omega_{0} \dot{y}_{0}(t)+\left(\omega_{0}^{2}+\frac{c_{1} k_{a} k_{c}}{M}\right) y_{0}(t) \\
&-c_{3} \dot{y}_{0}\left(t-h_{1}\right)-2 \xi \omega_{0} c_{3} \dot{y}_{0}\left(t-h_{1}\right)-\left(\omega_{0}^{2} c_{3}\right. \\
&\left.+\frac{c_{2}}{M}\right) y_{0}\left(t-h_{1}\right)=0
\end{aligned}
$$

and the corresponding characteristic polynomial would be

$$
\begin{aligned}
c(s, z)=s^{2}+ & 2 \xi \omega_{0} s+\left(\omega_{0}^{2}+\frac{c_{1} k_{a} k_{c}}{M}\right)-c_{s} s^{2} z \\
& -2 \xi \omega_{0} c_{s} s z-\left(\omega_{0}^{2} c_{3}+\frac{c_{2}}{M}\right) z=0
\end{aligned}
$$

where the gains $k_{a}, k_{b}$, and $k_{c}$ are all assumed to be positive. The following theorem is a direct application of the analytic stability test of Theorem 2.

Theorem 4 Consider the multipass process (29) and assume positive gains $k_{a}, k_{b}$, and $k_{c}$. Then the commensurate metal rolling process is asymptotically stable i.o.d. if and only if

$$
\begin{aligned}
& \text { (i) } k_{a}>\frac{c_{2}-\lambda\left(1-c_{3}\right)}{\left(k_{c} \lambda / \lambda_{2}\right)+1-c_{3}} \\
& \text { (ii) } k_{a} k_{c}<\lambda_{1}+\frac{\lambda_{1}\left(1-c_{3}^{2}\right)}{2 c_{2} M} k_{b}^{2}
\end{aligned}
$$

Proof: The proof of this theorem can be developed based on theorem 2.
Hence, the inequalities (31) and (32) give the design constraints on the $\mathrm{PD}$ controllers gains to guarantee the asymptotic stability i.o.d. for the controlled commensurate metal rolling process.

\section{Discussion and Conclusions}

The metal rolling process has been modelled using delay differential equations with commensurate and noncommensurate delays. These delays correspond to pass delay and measurement delays. It has been demonstrated that the theory of multidimensional systems, delay differential or multipass systems, is a natural setting for the study of the metal rolling process. It has been shown

that the conditions of asymptotic stability i.o.d. can be employed to calculate bounds for the controlled process controller gains to guarantee stability against delay variations.

\section{Acknowledgment}

This work has been supported by NSERC

\section{References}

[1] L.E. Doyle et al., Manufacturing Processes and Materials for Engineers, Prentice-Hall, 1985

[2] J.B. Edwards, "Stability Problems in the Control of Multipas Proceses", Proc. IEE, Vol. 121, No. 11, pp. 1425-1432, 1974

[3] J.B. Edwards, 'Wider Application of Multipass Systems Theory, Pt. 1: Multimachine and Multicell Systems", Proc. IEE, Vol. 125, No. 5, pp. 447-452, 1978

[4] J.B. Edwards, "Wider Application of Multipass Systems Theory, Pt. 2: Controlled Distributed processes", Proc. IEE, Vol. 125, No. 5, pp. 453-459, 1978

[5] J.B. Edwards and D.H. Owens,: Analyois and Control of Multipass Processes Research Studies Press, Chichester, 1982

[6] D.H. Owens, "Stability of Linear Multipass Processes", Proc. IEE, Vol. 124, No. 11, pp. 1079-1082, 1977

[7] D.H. Owens, 'Asymptotic Stability of Differential Multipass Processes", Electronics Letters, Vol. 15, No. 15, pp. 446-447, 1979

[8] F.M. Boland and D.H. Owens, "Linear Multipass Processes: A Two-Dimensional Interpretation ${ }^{»}$, Proc. IEE, Pt. D, Control Theory \& Appl., Vol. 127, No. 5, pp. 189-193, 1980

[9] S. Foda and P. Agathoklis, "On the Stability of Differential Multipass Processes", Submitted for publication.

[10] J. Chiasson and E.B. Lee, "Control of Multipass Processing Using 2-D System Theory", Proc. IEEE Conf. on Decision \& Control, Ft. Lauderdale, FL, pp. 17341737,1985

[11] S. Foda, Stability and Stabilization of Multidimensional Systems, Ph.D. Dissertation, Department of Electrical \& Computer Engineering, University of Vic- 
toria, 1988

[12] D. Herts, E.I. Jury, and E. Zeheb, "Simplified Analytic Stability Test for Systems with Commensurate Delays", Proc. IEE, Pt. D, Control Theory \& Appl., Vol. 131, No. 1, pp. 52-56, 1984

[13] A. Hmamed, "Stability Conditions of Delay-Differential Systems", Int. J. Control, Vol. 43, No. 2, pp. 455-463, 1986

[14] X-Y. Liu and M. Mansour, "Stability Test and Stability Conditions for Delay Differential Systems", Int. J. Control, Vol. 39, No. 6, pp. 1228-1242, 1984

[15] P. Agathoklis and S. Foda, "Stability and the Matrix Lyapunov Equation for Delay Differential Systeme", To appear in Int. J. Control.

[16] S.H. Żak, E.B. Lee, and W-S. Lu, "Realization of 2-D Filters and Time Delay Syatems", ILED Trans. Circuits and Syateme, Vol. CAS-33, No. 12, pp. 1241-124, 1986

[17] C.I. Byrnes, M.W. Spong, and T.J. Tarn, "A Several Complex Variable Approach to Feodback Stabiliration of Linear Neutral Delay-Differential Systems," Math. Syat. Theory, Vol. 17, pp. 97-133, 1984

[18] M.W. Spong, "A Semistate Approsch to Feedbeck Stwbilization of Neutral Delay System", Cireuits, Sytems, \& Signal Procese., Vol. 5, No. 1, pp. 69-85, 1986

[19] J. Hale, Theory of Fuctional Differential Equations, Springer-Verlag, New York, 1977

[20] S. Foda and P. Agathollis, "Aymptotic Stability of Delay Differential Systems with Noncommensurate Delays", Submitted for publication.

[21] R.D. Driver, Ordinery end Deley Differential Equetiom, Springer-Verlas, New York, 1977

[22] M. Crus and J. Hale, "Stability of Functional Differential Equations of Neutral Type", J. of Dif. Equations, 7, Pp. 334-355, 1970

[23] R.E. Kamen, "On the Relationship Between Zero Criteris for Two-Variable Polynomiala and Aymptotic Stability of Delay-Differential Equations", IDED Trans. Automatic Control, Vol. AC-25, No. 5, pp. 983984, 1980

[24] R.E. Kamen, P.P. Khargonetar, and A. Tannenbaum, "A Local Theory of Livear Systems With Noncommensurate Time Delays", Proc. MTNS-83, Lecture Notes in Control and Information Sciences, Vol. 58, SpringerVerlag, Berlin, 1984

[25] S. Barnett, Polynomiale and Linear Control Syotems, Marcel Dekker, New York, 1983

[26] B.D.O. Anderson and S. Vongpanitlerd, Network Arelyois and Syntheris, Prentice-Hall, Inc., Bnglewoods Cliffs, New Jersey, 1973

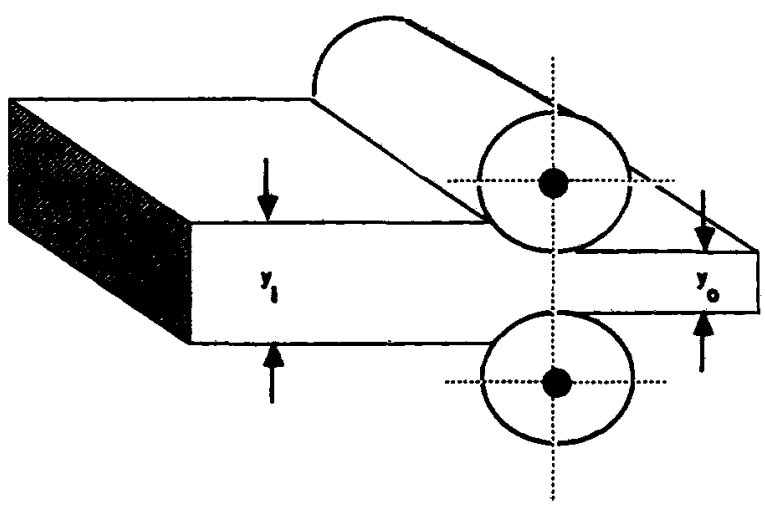

Figure 1: Schematic Representation of Longitudinal Rolling

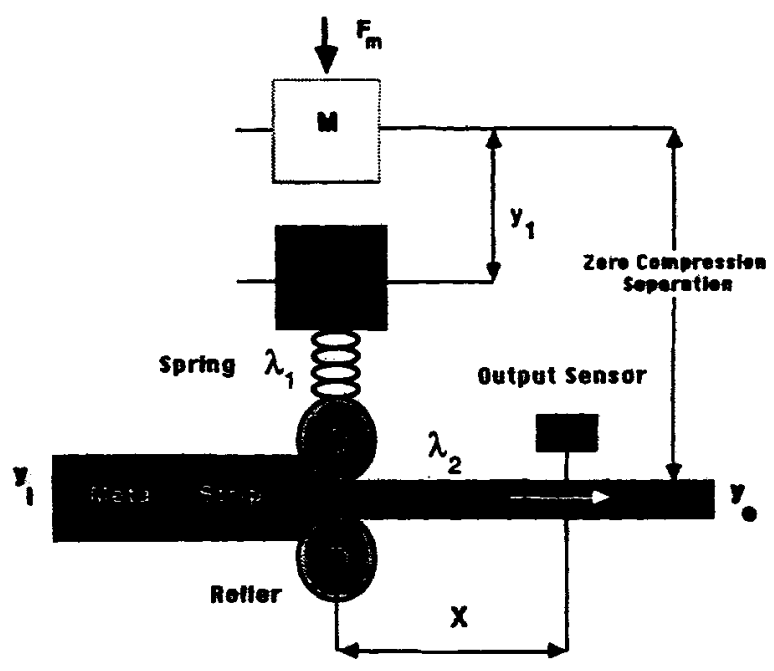

Figure 2: Physical Blagrem of the Metat nolHng Process
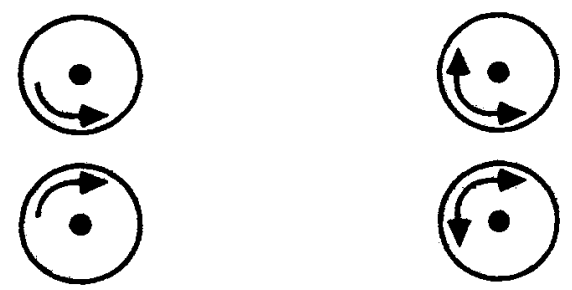

Non-reversing

Reversing

Figure 3: Twe Migh Rolling Stends 